\title{
The "Out-of-the-Loop" concept in automated driving: proposed definition, measures and implications
}

\author{
Natasha Merat ${ }^{1}\left(\mathbb{D} \cdot\right.$ Bobbie Seppelt $^{2} \cdot$ Tyron Louw $^{1} \cdot$ Johan Engström $^{3} \cdot$ John D. Lee $^{4} \cdot$ Emma Johansson $^{5}$. \\ Charles A. Green ${ }^{6}$. Satoshi Katazaki ${ }^{7}$. Chris Monk ${ }^{8} \cdot$ Makoto Itoh $^{9} \cdot$ Daniel McGehee $^{10} \cdot$ Takashi Sunda $^{11}$. \\ Kiyozumi Unoura ${ }^{12} \cdot$ Trent Victor $^{13} \cdot$ Anna Schieben $^{14} \cdot$ Andreas Keinath $^{15}$
}

Received: 28 April 2018 / Accepted: 29 August 2018 / Published online: 15 September 2018

(c) The Author(s) 2018

\begin{abstract}
Despite an abundant use of the term "Out of the loop" (OOTL) in the context of automated driving and human factors research, there is currently a lack of consensus on its precise definition, how it can be measured, and the practical implications of being in or out of the loop during automated driving. The main objective of this paper is to consider the above issues, with the goal of achieving a shared understanding of the OOTL concept between academics and practitioners. To this end, the paper reviews existing definitions of OOTL and outlines a set of concepts, which, based on the human factors and driver behaviour literature, could serve as the basis for a commonly-agreed definition. Following a series of working group meetings between representatives from academia, research institutions and industrial partners across Europe, North America, and Japan, we suggest a precise definition of being in, out, and on the loop in the driving context. These definitions are linked directly to whether or not the driver is in physical control of the vehicle, and also the degree of situation monitoring required and afforded by the driver. A consideration of how this definition can be operationalized and measured in empirical studies is then provided, and the paper concludes with a short overview of the implications of this definition for the development of automated driving functions.
\end{abstract}

Keywords Out of the Loop · Automated driving $\cdot$ Autonomous $\cdot$ Driver behaviour $\cdot$ Human factors

Natasha Merat

n.merat@its.leeds.ac.uk

1 Institute for Transport Studies, University of Leeds, Leeds, UK

2 MIT Center for Transportation and Logistics, Cambridge, MA 02139, USA

3 Virginia Tech Transportation Institute, Blacksburg, VA 24061, USA

4 Industrial and Systems Engineering, 1513 University Avenue, Madison, WI, USA

5 Volvo Group Trucks Technology, Gothenburg, Sweden

6 General Motors Co., Detroit, USA

7 National Institute of Advanced Industrial Science and Technology (AIST), Tsukuba, Japan
8 National Highway Traffic Safety Administration, Washington, DC, USA

9 Faculty of Engineering, Information and Systems, University of Tsukuba, Tsukuba, Japan

10 Mechanical and Industrial Engineering and National Advanced Driving Simulator, University of Iowa, Iowa City, USA

11 Nissan Motor Corporation Ltd., Yokosuka, Japan

12 Honda R\&D Co., Tokyo, Japan

13 Volvo Cars, Gothenburg, Sweden

14 DLR German Aerospace, Brunswick, Germany

15 BMW Group, Munich, Germany 


\section{Introduction and background}

This article summarizes the discussions and conclusions of members of the Trilateral Human Factors Working Group, tasked by a high-level agreement between Europe, North America and Japan to share knowledge and exchange findings from studies on the human factors challenges and opportunities of vehicle automation.

The paper aims to provide a precise definition, and conceptual framework, for the term "Out of the Loop" (OOTL), which has been used liberally in recent literature, when considering the effect of road vehicle automation on driver behaviour and performance. It is commonly suggested, but currently without concrete evidence, that increasing levels of automation likely reduce driver interactions with, and control of, certain aspects of the driving task, which can compromise drivers' ability to intervene and respond to safety-critical events in a timely and appropriate manner. This is based on the assumption that simply removing drivers from direct physical control of the vehicle may leave them "Out of the Loop" when it comes to such responses. However it is not currently clear what the OOTL term refers to with regards to human information processing and response capabilities, how the term relates to other constructs in the human factors/behavioral sciences literature, how it can be measured, and, ultimately, how and whether it affects driver performance and safety.

Therefore, the task of group members was to consider the following questions, which were discussed in a series of face to face and on-line meetings:

1. How can being in the loop be precisely defined?

2. How can being in the loop be measured?

3. What are the likely implications of being out of the loop?

An initial observation following our discussions was that, even among the team of Human Factors professionals assembled to define OOTL, there were fundamental differences in how the "the loop" was defined, which prompted the need for a precise and commonly-agreed definition, to determine whether the driver is "in" or "out" of such a loop. We also agreed that it was important to distinguish between situations that induce an OOTL state, versus the consequences of this state on driving performance. Finally, when defining OOTL, and identifying a set of measures for determining when a driver was in this state during the use of different types and levels of automation, it is important to appreciate the manufacturers' intended use of an automated system, and whether or not the human driver understands its overall capabilities and limitations, and is expected to supervise its operation.
Following an agreement amongst the authors of this paper, about the responsibilities and requirements of humans during the manual task of driving, a synthesized framework for defining OOTL has emerged from these individual perspectives, and will be outlined further below. This framework begins with the role of drivers, and the tasks they perform during use of an automated system and distinguishes between being in, on or out of the loop.

It is hoped that this shared understanding of the OOTL concept among those working in the field from the three regions can (1) be used to study the concept and its consequences more precisely, (2) identify gaps in the research in this area, and (3) highlight the likely (negative and positive) consequences of a driver who is 'Out of the Loop' during use of automated systems in a road vehicle. This shared understanding will also allow practitioners, technology manufacturers, policy makers, and regulators, to appreciate the likely consequences of different types of road vehicle automation on driver performance and safety. Reaching agreement on a shared definition of the concept, and how it can be measured, is expected to help researchers compare the outcomes from studies in this area, easing interpretation of research findings in different experimental contexts. Such an understanding should also promote the design of ecologically-valid and relevant studies-ones designed to appreciate the likely human factors implications, challenges, and benefits of road vehicle automation.

We begin with a short overview of previous work related to defining the OOTL concept, including its origin, and how it has been used in other domains involving human interaction with automation. This includes a set of concepts from the driver behaviour and human factors literature, which are then used as the basis for our novel definition of OOTL. We conclude by providing a brief outline of how OOTL, as defined here, can be measured, and discuss the implications of our OOTL definition when considering the design of automated driving functions.

\section{Current use of the Out of the Loop (OOTL) concept: the need for a common definition}

One of the earliest references to the term "Out of the Loop" is found in a 1972 US patent filed by Willard Meilander of Goodyear Aerospace Corporation for a series of methods and systems used for automated aircraft control (Meilander 1972). The patent describes situations where "the controller could be left out of the loop and the plane or planes involved could be directed by the computer", but offers no further definition. Research conducted in the 1970s and 1980s, examined the consequence of leaving the controller out of the loop and found that, perhaps due to a reduced role, pilots were slower to detect changes in control dynamics 
and did not recover as well as those who remained in the manual control loop (Wickens and Kessel 1979, 1981; Wickens 1992). Though there are references to the term in this literature, Endsley and Kiris (1995) and Kaber and Endsley (1997) were the first to formalize the concept. Drawing on studies investigating the performance of pilots and control systems, with operators using systems capable of varying degrees of automation, Kaber and Endsley (1997) argue that "When an operator is removed from a control loop due to allocation of system functions to an automated/computer controller, the level of human-system interaction is limited and, consequently, operator awareness of system states may be reduced". In such situations, operators are more likely to make errors when interacting with, or when taking control from, the system (see Sebok and Wickens 2017, for a recent overview).

Linked to this original reference in the aviation domain, the term "loop" likely originates from the "open- and closedloop" concepts described by Control Theory in Systems Engineering. For example, "loop" is often used in engineering contexts as a shorthand for "control loop" and refers to elements of a system, and their connections, which are involved in the control of that system. In addition to its use in the engineering field, "control" is also a term used in the behavioral sciences/psychology domain, where controlled performance relies on "higher" cognitive functions, such as working memory; requires attentional effort; and is needed to deal with novel or inherently difficult tasks. This is often contrasted to automatic performance which is effortless, not available to consciousness and thought to be established through repeated exposure to consistent mappings between stimuli and responses (Schneider and Shiffrin 1977). In both fields, "control" describes the process of sensing and responding to changes in the environment to achieve a goal state, or to reduce uncertainty, and, regardless of domain, this "control" requires some form of interaction that acts on input to affect the state of a system. Although a precise definition of control is provided by both engineering and the behavioral sciences, these have not been integrated and reconciled in describing situations where humans and automation jointly control a process, which is important when considering the relationship between the driver and vehicle in automated driving.

Common among previous references to OOTL for onroad motor vehicles is removal of the human from physically controlling the vehicle (e.g., Brookhuis et al. 2001). Here, there is a fundamental shift of the driver's role from a physical controller to a supervisor, who may now be monitoring the driving task and automated systems (e.g., Saffarian et al. 2012; Strand et al. 2014). On the other hand, some authors use of "Out of the Loop" to refer more specifically to instances when drivers are not actively monitoring the system and the traffic situation (e.g. Radlmayr et al. 2014; Merat et al. 2014; Casner et al. 2016; Louw and Merat 2017). Links are also made in the literature between being OOTL and daydreaming or distraction (e.g. Norman 2015; Berberian et al. 2017).

Thus, the distinction between physical and cognitive aspects of control plays a key role in discussions about OOTL, in which the loss of physical control generally refers to taking the hands away from the steering wheel or the foot off the pedals, whereas the loss of cognitive control typically refers to taking attention away from the main driving task (Louw et al. 2015a, b). However, it is not always clear if the cognitive aspect/attentional element referenced pertains specifically to cognitive control (i.e., effortful, conscious, focusing of attention, resulting in controlled performance), or, more generally, to control that requires some degree of cognition beyond the physical control of the vehicle. The link between such aspects of cognitive control and monitoring the driving task and environment is also not clear.

In line with Kaber and Endsley's (1997) definition, the International Harmonized Research Activities (IHRA) Working Group on Intelligent Transport Systems (ITS), supporting the activities of the United Nations Economic Commission for Europe (UNECE) Work Package 29 (World Forum for Harmonization of Vehicle Regulations), defined a driver as being OOTL when they are "not immediately aware of the vehicle and the road traffic situation because they are not actively monitoring, making decisions or providing input to the driving task" (Kienle et al. 2009). Here, again, the key concepts "aware", "monitoring", "decisions" and "input" can be linked to both cognitive and physical aspects of the loop, but are not explicitly defined. Moreover, this definition does not elaborate on the multi-level nature of the driving task, nor does it consider the likely consequences of being OOTL.

The next section, therefore, develops a more precise definition of OOTL, by considering a set of concepts from the human factors and driver behaviour literature, which includes a consideration of the multifaceted nature of driving and an overview of findings linked to driver OOTL state.

\section{Defining "Out of the Loop" in terms the control loops and attention in driving}

As outlined above, the term "Out of the Loop" has been used inconsistently within academic literature in reference to the physical and cognitive aspects of control. We suggest that three broad areas are important when considering the OOTL concept, which, when considered together, allow us to orient and organize a common definition of "the loop". These include: (1) the relevance of loops in vehicle control; (2) attention, automaticity and control; and (3) perceptualmotor coupling and situation awareness. 
Following this overview, we discuss how and when the addition of system automation might reduce or remove human involvement in "a loop", and promote involvement in "another loop", and whether or not this transfer impairs performance, and reduces safety.

\subsection{The relevance of loops in vehicle control}

A hierarchical control structure is typically referenced when defining vehicle control in the driving task (Allen et al. 1971; Hollnagel and Woods 1995; Michon 1985; Ranney 1994). Figure 1 shows the multi-level hierarchical control tasks or functions of driving. Each represents a different control loop. In the innermost loop, drivers perform the momentto-moment (ms-s), or continuous, lateral and longitudinal motion control tasks, initiating braking/accelerating and steering actions, to maintain/adjust speed as well as position the vehicle within a lane; these are the operational functions of driving. In the middle loop, drivers perform the intermittent (s-min) object/event detection and response tasks, here representing planning and execution of maneuvers such as lane changes, and negotiating intersections; these are the tactical functions of driving. In the outermost loop, drivers select destinations and perform waypoint finding along a route in the infrequent (min-h) task of navigation; these are the strategic functions of driving.

For the present purposes, two aspects of this hierarchical representation of driving are of key importance. First, the levels of the hierarchy are essentially defined by the spatiotemporal scale of the different driving subtasks. Drivers initiate millisecond-to-second operational control of the steering wheel and pedals, both in the service of maintaining lateral and longitudinal position within a lane, and in response to objects and events. In response to the characteristics of the driving environment, the subtask of "object event detection and response (OEDR)" can vary in its temporal frequency from a continuous (ms-s) to an infrequent (s-min) activity. Maintaining a safe headway to a lead vehicle in heavy or stop-and-go traffic, or steering on a curvy mountainous road, are two example situations in which object/event detection and response can occur almost continuously, compared to control of a vehicle on a straight, no traffic, rural road.

Second, in manually controlled vehicles, subtasks at different levels are strongly interdependent. Thus, the driver's physical engagement through braking, accelerating, and steering activities is not linked to a particular loop. A driver's moment-to-moment operational control in the amount of steering input and level of braking/accelerating affects the vehicle's movement, relative to other vehicles and its position within the lane, which affects the potential for encountering obstacles and hazards in the driving environment. In turn, how drivers maneuver within a lane, and in response to detected objects or roadway events, affects potential path changes needed to successfully perform the task of navigating. Goals for navigation can in turn influence how and when drivers initiate maneuvers, such as lane changes and turns, which in turn impacts how drivers move relative to other vehicles and with respect to lane boundaries. In this sense, the control loops in Fig. 1 are meant to provide a simplistic subtask categorization that duly depicts, through its nested hierarchical structure, the more complex functional interdependencies that exist in a description of the physical control involved in the driving task. This representation, however, neglects to explicitly represent the flow of information in the driver's interaction with the vehicle while moving through a dynamic environment, which is linked to aspects of both automatic and controlled cognition, described further in the next section.

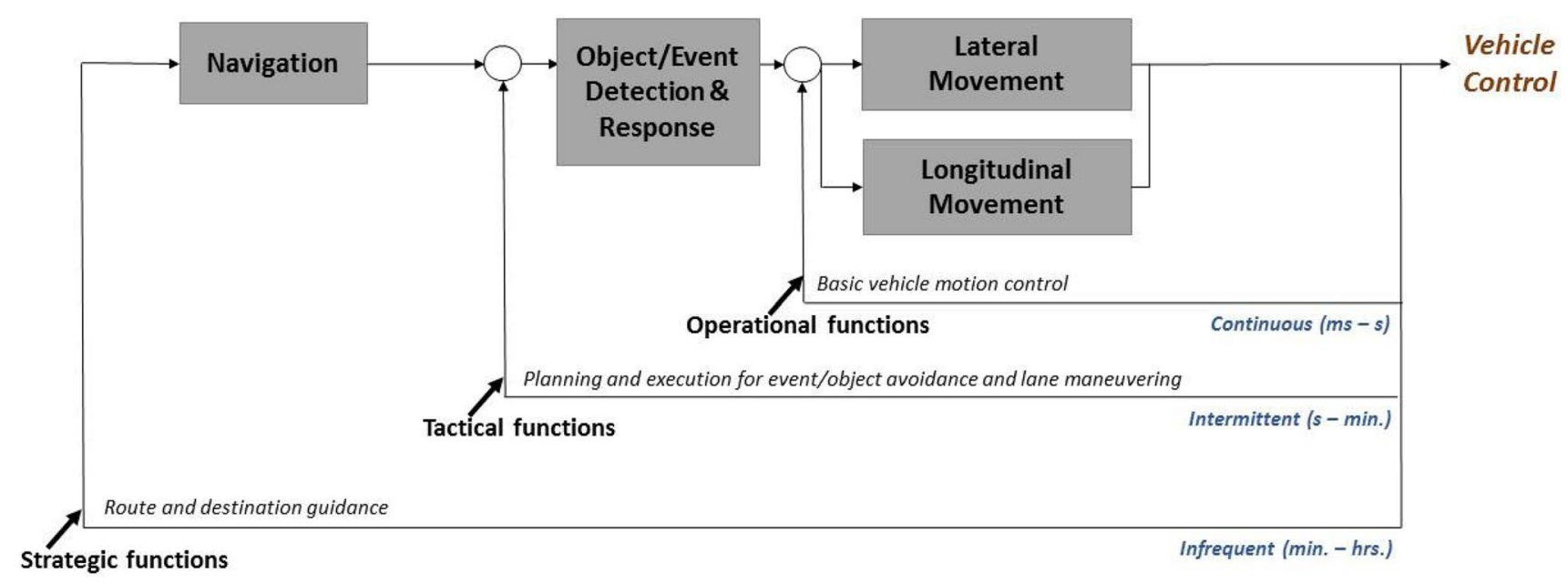

Fig. 1 Multi-level control in driving [based on Michon's (1985) levels; adapted from Fig. 1 in SAE (2016a, b)] 


\subsection{Attention, automaticity, and control}

In the interplay of the driver with the vehicle and environment, sensory information guides control. To perform the control tasks of driving, a driver must attend to the forward view at regular intervals. Driving is primarily a visual task (e.g., Michaels 1963), and visual information is mostly used to maintain basic vehicle control, to detect cues in the environment that signal potential hazards and obstacles, and to orient to potential or impending changes in the route. The rate of visual sampling required to maintain vehicle control depends largely on the rate by which information changes (Senders et al. 1967). At higher speeds, and with increased information density of the roadway-which can be a function of traffic, roadway curvature, lane width, and roadway layout (McDonald and Ellis 1975; Tsimhoni and Green 2001; Tivesten and Dozza 2014)_drivers must sample the forward view more frequently to maintain position within the lane. In addition to visual cues, drivers make use of proprioceptive and auditory feedback while controlling the vehicle in their movement through the roadway environment. Feedback provided via in-vehicle interfaces additionally informs on vehicle state and on-board system status.

A key aspect of control (at all hierarchical levels) is attention, broadly referring to the selection of some aspects of a situation or task over others. However, there is a lack of consensus on the precise meaning of attention, both in general cognitive science/human factors research, and in the driver behaviour literature. To tackle this issue, work previously conducted by the bilateral (US-EU) predecessor to the present Trilateral Human Factors Working Group attempted to provide a more precise definition of attention and inattention in the context of driving (Engström et al. 2013). These authors argue that, while attention is often considered in terms of the selection of perceptual information, for applied/everyday tasks such as driving, it is useful to view driver attention more broadly in terms of the allocation of resources to a set of activities, thus also including the selection of action in the scope of attention. By the same token, driver inattention can be considered a mismatch between the current allocation of attentional resources, and those resources demanded by activities critical for safe driving (Lee et al. 2009).

The term resource is traditionally associated with limited processing capacity and mental effort (e.g., Broadbent 1958; Kahneman 1973). However, the resource concept here is used in a broader sense to denote any sensory, actuator, perceptual, motor or cognitive mechanisms that are used in performing activities (Wickens 2002). Hence, by contrast to its traditional meaning, resources may also include mechanisms underlying automatized performance of routine activities. Sensory resources include sense organs such as the eyes or the ears, while actuator resources refer to parts of the body used to control devices (steering wheel/pedal), such as the hands or the feet. Perceptual resources refer to neural mechanisms underlying detection and interpretation of information, and motor resources to neural mechanisms that control overt action. Finally, cognitive resources refer to neural mechanisms underlying cognitive control, which relates to working memory and the effortful deployment of resources to deal with non-routine or novel tasks.

In this context, it is important to have a common agreement on whether terms such as "attention" and "resources" refer to controlled (i.e., relying on cognitive/executive control) or automatic (not requiring conscious control) performance. At least in routine driving situations, it can be argued that many, if not most, activities are likely to be more or less automatized, for experienced drivers. Therefore, it is important to establish whether being in or on the loop requires an element of controlled attention, or whether automatized use of resources is sufficient.

\subsection{Perceptual-motor coupling and Situation Awareness}

When defining "the loop" (and in determining how to measure it), it is important to recognize the dependency that exists between the physical and cognitive aspects vehicle control, and the relationship between this perceptual-motor coupling and driver's situation awareness. Figure 2 shows how the driving task invokes a continuous processing of perceptual information, and that monitoring is inherent at multiple levels in driving. Implicitly built into the hierarchical control loops that describe the functional tasks of driving (Fig. 1) is the driver's role in monitoring the environment. For manual driving, vehicle control and monitoring should go hand in hand, although lapses in the latter can occur (see below). In moving through a dynamic environment, drivers must adapt their monitoring of information to safely perform the driving task. Perceptual-motor coupling in manual driving involves regular sampling between a distant, far-road, region and a close, near-road region (Land and Horwood 1995; Salvucci and Gray 2004) to modulate the steering wheel and pedals for operational lane-keeping and headway control. The sampling, modulation of controls, and sensing of the control inputs are tightly coupled. A moment-to-moment perceptualmotor cycle generates the predictions of surrounding traffic dynamics and changes in the roadway and environment (e.g., Regan et al. 2009). Importantly, the perceptual cues used for vehicle control are not only visual but also include, for example, somatosensory, kinesthetic, proprioceptive and auditory cues.

To maintain situation awareness, drivers distribute their gaze across multiple on-road regions to safely perform moment-to-moment control (e.g., Victor et al. 2008). Gaze distributed across central and peripheral regions of 


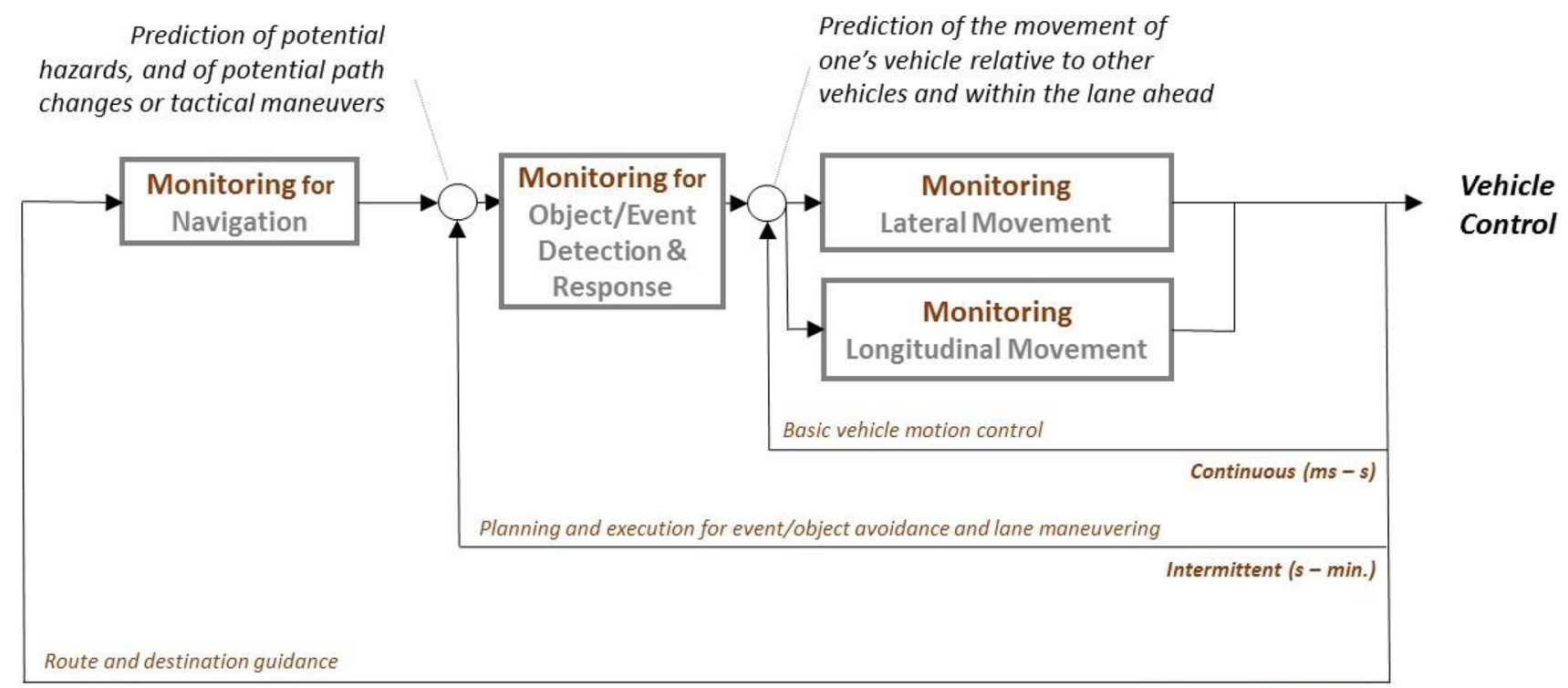

Infrequent (min. - hrs.)

Fig. 2 Monitoring inherent to multi-level control in driving

the roadway enables drivers to detect and identify hazards (Lamble et al. 1999; Samuel and Fisher 2015), to perform tactical maneuvers such as lane changes, turns at intersections (Harbluk et al. 2007), and to identify relevant signs and landmarks for route orientation and navigational tasks.

A useful concept used to characterize monitoring in terms of drivers' attention to the meaning of dynamic changes in their environment is Situation Awareness (SA), defined as "the perception of the elements in the environment within a volume of time and space, the comprehension of their meaning, and the projection of their status in the near future" (Endsley 1995, p. 36). A higher level of SA-that in which projection of environmental state occurs-depends upon perception and comprehension of relevant cues. The process of developing and maintaining SA iterates at the same timescales as driving; all levels of SA are involved in each of its three control loops (Ma and Kaber 2006). SA influences decision-making; subsequent actions change the state of the environment, which in turn influences a new cycle of perception; this cycle is illustrated in Fig. 3 (adapted from Ma and Kaber 2005).

\subsection{Defining 'Out-of-the-Loop'}

Considering the above concepts together, we suggest that "being in the loop" can be understood in terms of (1) the driver's physical control of the vehicle, and (2) monitoring the current driving situation. Vehicle physical control implies a direct physical coupling between multisensory perceptual cues and motor outputs (steering, accelerating/braking) and incorporates both operational and tactical functions of driving: maintaining adequate lateral and longitudinal vehicle position and executing lane-changing maneuvers.

As discussed above, situation monitoring can be characterized in terms of situation awareness, that is, drivers' comprehension of dynamic changes in their environment, including those related to the actions of an automated system. Thus, merely attending (i.e., allocating resources) to driving is not sufficient. To be adequately monitoring the situation, one must attend to and understand how the situation will develop. Hence, perception-action coupling and situation monitoring, as conceived here, can be viewed as closely related and reflecting the same basic phenomenon at different levels of the driving task. Thus, when in the loop, the driver can be seen as dynamically "embedded" in driving, perceiving the past, comprehending the present and predicting the future at different levels of the driving task.

Conversely, being "out of the loop" implies the lack of physical vehicle control and/or a lack of situation monitoring. We further suggest that in a situation where physical vehicle control is taken over by an automated system, the driver may still be regarded as being on the loop if $s($ he) is still engaged in situation monitoring. Based on these concepts, in-, on- and out of the loop may be defined as follows:

In the loop In physical control of the vehicle and monitoring the driving situation

On the loop Not in physical control of the vehicle, but monitoring the driving situation

Out of the loop Not in physical control of the vehicle, and not monitoring the driving situation, OR in physical control of the vehicle but not monitoring the driving situation 


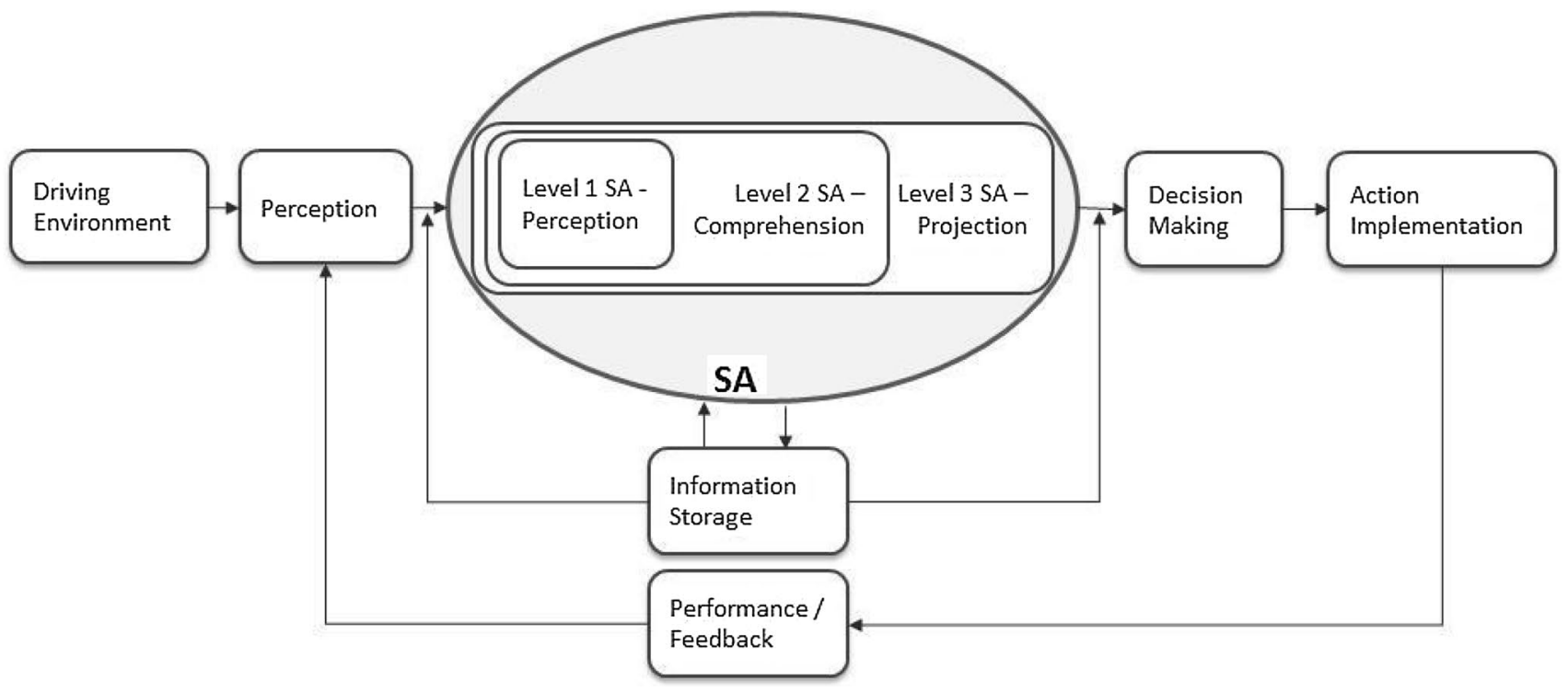

Fig. 3 SA in driving information processing (adapted from Ma and Kaber 2005)

In these definitions, the "driving situation" being monitored not only refers to the surrounding driving environment but also, potentially, to the actions of an automated system, and its current state. Furthermore, being in-, on- and out of the loop should not be viewed as discrete states, but rather levels of engagement along a continuum. This may, for example, apply in situations where an automated system is responsible for performing the steering task, but provides continuous force feedback to the driver, thus still keeping the driver in the lateral control loop, to some degree.

Moreover, the definitions do not imply that effortful, conscious, focusing of attention, that is, cognitive control, is a necessary condition for being in the loop. The need for the cognitive control required for maintaining attention on a task varies on a moment-to-moment basis, and depends on the degree of practice and familiarity with the current task or situation. Thus, being in the loop, that is, being successfully engaged in physical vehicle control and monitoring, may require cognitive control in novel or uncertain situations, but may proceed more automatically in other, familiar and less complex, scenarios. The automatic versus (cognitively) controlled performance dimension should also be viewed as a continuum rather than a discrete, binary, phenomenon.

Based on the above concepts, there is potential for the degree of physical vehicle control and monitoring to be reduced when some aspects of control are transferred from manual to automated driving. First, the richness of multisensory cues used for physical vehicle control is reduced during automated control (Wickens and Kessel 1981; Zuboff 2010). Second, monitoring could be compromised if the driver chooses to engage in non-driving related tasks (NDRTs) during driving automation (Norman 1990). Recent research has attempted to measure the consequences, if any, of such OOTL phenomena during automated driving. A short overview of this research is provided in the following section to help clarify how OOTL might be measured.

\section{Methods and measures for quantifying the OOTL concept}

The multi-faceted aspects of vehicle automation and associated levels of OOTL suggests a need for a correspondingly multi-faceted measurement approach. To date, most of the measures used for assessing changes in physical vehicle control and situation monitoring, at and after transition from automated driving, have focused on timing, and type of driver response, such as timing of hand(s) back on the wheel, or feet on the pedals, and/or timing, direction and force of steering, or ability, to successfully avoid colliding with obstacles (Gold et al. 2013, 2018; Zeeb et al. 2015, 2017; Eriksson and Stanton 2017; Louw et al. 2016, 2017). Therefore, drivers can be assumed to be OOTL, if, compared to manual driving, their response to impending collisions is impaired, for example due to a later or less efficient movement of the steering wheel, or a slower brake response time. A consideration of drivers' monitoring performance is also needed to confirm instances of "on" versus "out" of the loop.

In terms of drivers' situation awareness and monitoring of the environment, due to its ease of use, research has mostly relied on measuring drivers' visual attention to the driving situation, although some studies have also utilized neuro-imaging techniques for monitoring brain activity (Russel et al. 2016). In terms of eye-tracking, eye 
gaze and fixation patterns to different elements of the vehicle and the road are used to quantify "monitoring" and drivers' ability to detect and/or avoid impending or real obstacles and hazards after a period of automated driving (Zeeb et al. 2015; Hergeth et al. 2016; Louw and Merat 2016; Louw et al. 2016; Seppelt et al. 2017). Results show that driver eye movements are generally more dispersed as automation level increases, with less focus towards the road center and road environment, and, for instance, more focus towards secondary/non-driving related tasks (NDRTs, Carsten et al. 2012; Louw and Merat 2016).

Performance measures used to assess a driver's state of being "in", "on", or "out" of the loop will also depend on the particular driving situation, roadway characteristics, speed of travel, and the type of control required by the system or the driver. However, based on the above definition, it is reasonable to conclude that measures of driver OOTL can be divided into two main categories: (1) vehicle-based sensors that can assess the degree of driver physical control of the vehicle, during or immediately after automation; and (2) driver-based sensors and measures, to assess the level and degree of monitoring dedicated to the driving situation. As outlined above, the latter includes use of devices for collecting driver physiological states, which may either be physically connected to participants (e.g. measuring heart rate), or capable of collecting data remotely. An example of the latter includes the use of video cameras to assess position of drivers' eye, head, face, hand and seating position/posture in the vehicle (Subit et al. 2017). Analysis of this video-based data may be in real-time or post-hoc, and can be supported or supplanted by observation studies conducted by the researcher, such as the Wiener Farhprobe (see Chaloupka and Risser 1995), Notably, though, this technique is a more resource-intensive option. Finally, video, observation and questionnaire-based metrics can be used in this context to establish drivers' subjective evaluation of the consequences of being OOTL, which follows reduced physical control and monitoring. Using either concurrent or post-hoc analysis, such investigations can, for example, assess whether reduced physical control and monitoring are concomitant with an increased engagement in NonDriving Related Tasks (NDRTs); how driver trust, understanding, and complacency ratings of the automated system are affected; and whether there is a change in detection of failures, or impairments in a driver's ability to respond to such failures (skill decrement) as a result of introduced automation.

In their overview of the multiple potential effects of automation, primarily incorporating work from the aviation domain, Seppelt and Victor (2016, pp. 142-143) provide a summary of the possible consequences of vehicle automation, and how these can be linked to loss of physical control/reduced monitoring of the driving situation. These include:

- Inaccurate or incomplete expectations of system response and behaviour, i.e., inability to anticipate situations that lie beyond the capabilities of the automation (Louw et al. 2016).

- Passive monitoring and failure to sample safety-critical areas such as cross-walks at intersections or glances to rear-view mirror, side mirror, indication with turn signals, and over-the-shoulder glances prior to lane changes (Gold et al. 2013; Louw et al. 2016).

- Increased uptake of secondary and non-driving related tasks (Carsten et al. 2012).

- Unnoticed mode transitions, for example from one level of automation to another (Sarter and Woods 1995).

- Low situation awareness scores or loss of awareness of the state and processes of the system (Endsley and Kiris 1995).

- Lower self-reported scores in confidence to make decisions (or control vehicle manually) after system failure (Lee and Moray 1994).

- High trust scores and complacent reliance. Drivers' overtrust of the system, based on its capability for response, and their lack of monitoring and vigilance, based on an unjustified assumption of satisfactory system state. Overreliance on a driving automation system is sometimes termed complacency when it results from trusting a system more than is warranted (Parasuraman and Manzey 2010).

- Inaccurate mental models (as measured subjectively by testing knowledge of actions and limits of the system, i.e., its boundary conditions). Note: Operators with substantial previous experience and well-developed mental models detect disturbances more rapidly than operators without this experience (Wickens and Kessel 1981; Naujoks et al. 2017).

Further research is required, for automated vehicles in particular, to assess whether the above findings are valid, particularly in real-world conditions. Further knowledge on how behaviour changes in the longer term, after use of automated systems, is also important. To characterize if a driver is in, on, or out of the loop, measures that assess both physical control, and the level, and degree of, monitoring dedicated to the driving situation are needed as part of future research.

\section{Relevance of defining the OOTL concept to system designers}

As outlined above, in addition to providing an agreed-upon definition of the OOTL concept, how it can be measured, and what its consequences may be, it is important to determine 
the relevance of the OOTL concept to system designers and vehicle automation users. This includes promoting a clear understanding by designers of how/whether reducing physical control of the vehicle, and driver monitoring of the situation, impairs performance immediately after a period of automation, and what strategies can assist in mitigating this impaired performance, if present (see Carsten and Martens 2018). Moreover, providing drivers with an understanding of the consequences of being OOTL is also important, to support safe interactions with the system.

With respect to the user, this understanding is also linked to what system designers instruct for the degree of driver involvement and engagement during automation: i.e. the degree of physical control and situation monitoring required and expected from the user. Two basic principles are considered relevant when attempting to match the system designer's expectation for the driver's involvement, versus a driver's actual involvement during different stages of automated driving. First, manufacturers have a right and a duty to specify the proper use of a driving automation feature (i.e. mode of a driving automation system-see SAE 2016b), which outlines the design intent for how a human user should use the equipment, including information regarding automation state. A key design specification is whether a human user's role is to monitor the environment and the operation of the automated feature, and how much physical control is necessary. Second, the role of Human Factors scientists is to investigate the potential for misuse or incorrect use of driving automation, specifically where a human user does not perform in the field in a way that the manufacturer may have specified in their design. Examples include failure to monitor the situation and the imperfect system, by engaging in NDRTs, or reduced/impaired physical vehicle control. The potential causes behind such unintended use can also be linked to inadequate or incorrect communication of guidelines for using the system (e.g. through customer literature, marketing materials) or due to system design affordances, and inaccurate user expectations or mental models.

In addition, the complexity of the above issues is enhanced because some (perhaps most) vehicles will be equipped with driving automation systems which have multiple modes of operation, in which the mode of operation can change (either by system or human action) during a trip. This includes the potential for system mode changes, where the design intent for the human user changes from unsupervised to supervised, and vice versa, during a trip, which changes the degree of monitoring, and, potentially, the physical control required. Here the driver benefits most if the appropriate state of the automation system is easily observable and understood, so it is clear to the driver when their role may change from being out-of-the-loop to on-theloop. How quickly this occurs also depends on the rate at which the information to be processed by the driver changes.
Additionally, discussion of any "out-of-the-loop" human factors issues is only relevant to driving automation systems where the manufacturer's design intent includes appropriate human physical control or adequate monitoring and supervision of that system and its state. Such Human Factors issues may include unintended use (insufficient supervision) in that mode, or may be found due to transitions into and out of that mode, to and from any non-automation modes, or to any automation modes which do not require human monitoring and supervision. If the manufacturer's design intent does not require human supervision of the driving automation system, or a mode of that system, then any performance issue by the system which results in reduced or loss of physical control and/or collision cannot be considered a human factors issue, and is therefore not within the scope of this paper.

As outlined above, for automated features where the driver is expected to supervise the system, or be responsible for momentary levels of physical engagement, driver state monitoring techniques are a potentially useful method for identifying and supporting the focus of the driver engagement in the loop (remaining on the loop in this case). However, for features where the driver is only expected to be receptive to a take-over request, with no supervision or monitoring of the system required, driver state monitoring is only useful for verifying driver presence and receptivity (e.g. driver is awake and/or can see/hear/feel the alert). Note, however, that in such circumstances and at higher levels of automation, the driver is intentionally taken OOTL (by design and based on a normative definition of how a system should behave). In practice, however, based on the descriptive reality of how a system actually behaves, the driver may need to be kept "on-the-loop", for example, by actively monitoring the system, to ensure they are receptive to requests to resume manual control of the vehicle. For higher levels of automation, especially, additional studies may provide helpful information to determine the extent to which a driver is able to effectively and safely transition from being "out of" to "in to" the loop, without an intervening need for being "on" the loop.

\section{Summary and conclusions}

As manufacturers, system designers and users of automated vehicles start to learn the capabilities and limitations of the level of automation engaged by various systems in their vehicle, it is important for all stakeholders to have a common understanding of the implications of such engagements. As the role of the driver moves from one of a physical controller of the system, to one that supervises and monitors the driving situation, to one that is no longer required to either monitor, control or supervise, but only respond to system limitations, it is important for all stakeholders to have a 
common understanding of how each type and level of automated system engaged changes the driver's roles and responsibilities, and whether/how this is affected by other factors such as road and driver type, as well as driving environment.

If manufacturers, policy makers and practitioners can all agree on how different levels and types of automated features affect driver engagement, what the consequences of disengagement might be, and how this message can be successfully conveyed to system designers and users, then the main aim of higher levels of automated systems, to increase driver comfort and safety, may be achieved more successfully.

This paper proposes a commonly agreed definition of the Out of the Loop (OOTL) concept for automated road vehicles, and highlights the major research gaps remaining in this domain. It is hoped that amalgamating the views of a number of principal human factors/behavioral scientists from academia with that of leading vehicle manufacturers/system designers provides an understanding from both a scientific and applied perspective. Offering an agreed, multidisciplinary, definition, which includes and considers the human factor in this rapidly developing technological area, may provide additional impetus for automated systems to be both designed, and used, appropriately, without undue expectations from either the designers or the users of the system. It is hoped that these definitions can be operationalized and measured in future empirical studies, and that system designers can use it to achieve an ultimate goal for automated systems: for the driver to know when and where they are in charge of the system, and when and where they can safely and successfully allow the system to keep them out of the loop.

Acknowledgements We would like to thank the following colleagues for contributing to the discussions of the trilateral working group throughout preparation of this manuscript: Myra Blanco, Hanna Bellem, Tatsuru Daimon, Ryuji Funayama, Yi Glaser, Daniel Glaser, Sebastian Hergeth, Andrea Sparko, Gerald Schmidt, and Florian Weber. The working group meetings were also supported by funds from FP7 and H2020 projects VRA and CARTRE, funded by the European Commission.

Open Access This article is distributed under the terms of the Creative Commons Attribution 4.0 International License (http://creativeco mmons.org/licenses/by/4.0/), which permits unrestricted use, distribution, and reproduction in any medium, provided you give appropriate credit to the original author(s) and the source, provide a link to the Creative Commons license, and indicate if changes were made.

\section{References}

Allen TM, Lunenfeld H, Alexander GJ (1971) Driver information needs. Highway Res Rec 366:102-115
Berberian B, Somon B, Sahaï A, Gouraud J (2017) The out-of-theloop brain: a neuroergonomic approach of the human automation interaction. Annu Rev Control 44:303-315

Broadbent D (1958) Perception and communication. Pergamon, London

Brookhuis KA, De Waard D, Janssen WH (2001) Behavioral impacts of advanced driver assistance systems-an overview. EJTIR 1(3):245-253

Carsten O, Martens MH (2018) How can humans understand their automated cars? HMI principles, problems and solutions. Cogn Technol Work. https://doi.org/10.1007/s10111-018-0484-0

Carsten O, Lai FC, Barnard Y, Jamson AH, Merat N (2012) Control task substitution in semi automated driving: does it matter what aspects are automated? Hum Factor 54(5):747-761

Casner SM, Hutchins EL, Norman D (2016) The challenges of partially automated driving. Commun ACM 59(5):70-77

Chaloupka C, Risser R (1995) Don't wait for accidents—possibilities to assess risk in traffic by applying the 'Wiener Fahrprobe'. Saf Sci 19(2-3):137-147

Endsley MR (1995) Toward a theory of situation awareness in dynamic systems. Hum Factor 37(1):32-64

Endsley MR, Kiris EO (1995) The out-of-the-loop performance problem and level of control in automation. Hum Factor 37(2):381-394

Engström J, Monk CA, Hanowski RJ, Horrey WJ, Lee JD, McGehee DV, Regan M, Stevens A, Traube E, Tuukkanen M, Victor T, Yang CYD (2013) A conceptual framework and taxonomy for understanding and categorizing driver inattention. European Commission, Brussels

Eriksson A, Stanton NA (2017) Takeover time in highly automated vehicles: noncritical transitions to and from manual control. Hum factors 59(4):689-705

Gold C, Dambock D, Lorenz L, Bengler K (2013) "Take over!"- -how long does it take to get the driver back into the loop? In: Proceedings of the human factors and ergonomics society 57 th annual meeting, pp 1938-1942

Gold C, Happee R, Bengler K (2018) Modeling take-over performance in level 3 conditionally automated vehicles. Accid Anal Prev 116:3-13

Harbluk JL, Noy YI, Trbovich PL, Eizenman M (2007) An onroad assessment of cognitive distraction: impacts on drivers' visual behavior and braking performance. Accid Anal Prev 39(2):372-379

Hergeth S, Lorenz L, Vilimek R, Krems JF (2016) Keep your scanners peeled: Gaze behavior as a measure of automation trust during highly automated driving. Hum Factors 58(3):509-519

Hollnagel E, Woods DD (1995) Joint cognitive systems: foundations of cognitive systems engineering. Taylor and Francis, Boca Raton

Kaber DB, Endsley MR (1997) Out-of-the-loop performance problems and the use of intermediate levels of automation for improved control system functioning and safety. Process Saf Prog 16(3):126-131

Kahneman D (1973) Attention and effort, vol 1063. Prentice-Hall, Englewood Cliffs

Kienle M, Damböck D, Kelsch J, Flemisch F, Bengler K (2009) Towards an H-Mode for highly automated vehicles: driving with side sticks. In: Proceedings of the first international conference on automotive user interfaces and interactive vehicular applications (AutomotiveUI 2009), Essen, Germany, pp 19-23

Lamble D, Laakso M, Summala H (1999) Detection thresholds in car following situations and peripheral vision: implications for positioning of visually demanding in-car displays. Ergonomics 42(6):807-815

Land M, Horwood J (1995) Which parts of the road guide steering? Nature 377(6547):339-340

Lee JD, Moray N (1994) Trust, self-confidence, and operators' adaptation to automation. Int J Hum Comput Stud 40(1):153-184 
Lee JD, Young KL, Regan MA (2009) Defining driver distraction. In: Regan MA, Lee JD, Young KL (eds) Driver distraction: theory, effects, and mitigation. CRC, Boca Raton, pp 31-40

Louw TL, Merat N (2016) Are you in the loop? Using gaze dispersion to understand driver visual attention during vehicle automation. Transp Res Part C 76:35-50

Louw T, Merat N (2017) Are you in the loop? Using gaze dispersion to understand driver visual attention during vehicle automation. Transp Res Part C Emerg Technol 76:35-50

Louw T, Kountouriotis G, Carsten O, Merat N (2015a) Driver inattention during vehicle automation: how does driver engagement affect resumption of control? In: 4th international conference on driver distraction and inattention (DDI2015), Sydney: proceedings. ARRB Group

Louw T, Merat N, Jamson H (2015b) Engaging with highly automated driving: to be or not to be in the loop? In: 8th international driving symposium on human factors in driver assessment, training and vehicle design, Salt Lake City, Utah, USA

Louw T, Madigan R, Carsten O, Merat N (2016) Were they in the loop during automated driving? Links between visual attention and crash potential. Injury Prev 23(4):281-286

Louw T, Markkula G, Boer E, Madigan R, Carsten O, Merat N (2017) Coming back into the loop: drivers' perceptual-motor performance in critical events after automated driving. Accid Anal Prev 108:9-18

Ma R, Kaber DB (2005) Situation awareness and workload in driving while using adaptive cruise control and a cell phone. Int J Ind Ergon 35(10):939-953

Ma R, Kaber DB (2006) Presence, workload and performance effects of synthetic environment design factors. Int J Hum Comput Stud 64(6):541-552

McDonald B, Ellis NC (1975) Driver workload for various turn radii and speed. Transp Res Rec 530:18-29

Meilander WC (1972) U.S. patent no. 3,668,403. U.S. Patent and Trademark Office, Washington, DC

Merat N, Jamson AH, Lai FC, Daly M, Carsten OM (2014) Transition to manual: driver behaviour when resuming control from a highly automated vehicle. Transp Res Part F Traffic Psychol Behav 27:274-282

Michaels RM (1963) Perceptual factors in car following. In: Proceedings of the second international symposium on the theory of road traffic flow. Organization for Economic Co-operation and Development, Paris, pp 44-59

Michon J (1985) A critical view of driver behavior models: what do we know, what should we do? In: Evans L, Schwing RC (eds) Human behavior and traffic safety. Plenum, New York, pp 485-520

Naujoks F, Purucker C, Wiedemann K, Neukum A, Wolter S, Steiger R (2017) Driving performance at lateral system limits during partially automated driving. Accid Anal Prev 108:147-162

Norman DA (1990) The 'problem' with automation: inappropriate feedback and interaction, not 'over- automation'. Philos Trans R Soc Lond Ser B Biol Sci 327(1241):585-593

Norman DA (2015) The human side of automation. In: Road vehicle automation 2. Springer, Berlin, pp 73-79

Parasuraman R, Manzey D (2010) Complacency and bias in human use of automation: an attentional integration. J Hum Factors Ergonom Soc 52(3):381-410

Radlmayr J, Gold C, Lorenz L, Farid M, Bengler K (2014) How traffic situations and non-driving related tasks affect the take-over quality in highly automated driving. In: Proceedings of the human factors and ergonomics society annual meeting, vol 58(1). Sage, Los Angeles, pp 2063-2067

Ranney TA (1994) Models of driving behavior: a review of their evolution. Accid Anal Prev 26(6):733-750

Regan MA, Young KL, Lee JD (2009) Introduction. In: Driver distraction: theory, effects, and mitigation. CRC, Boca Raton, pp 3-7
Russell HE, Harbott LK, Nisky I, Pan S, Okamura AM, Gerdes JC (2016) Motor learning affects car-to-driver handover in automated vehicles. Sci Robot 1(1):eaah5682

SAE (2016a) Taxonomy and definitions for terms related to driving automation systems for on-road motor vehicles. In: SAE standard J3016 201609. SAE International, Warrendale

SAE (2016b) Human factors definitions for automated driving and related research topics. In: SAE standard J3114 201612. SAE International, Warrendale

Saffarian M, de Winter JC, Happee R (2012) Automated driving: human-factors issues and design solutions. In: Proceedings of the human factors and ergonomics society annual meeting, vol 56(1). Sage, Los Angeles, pp 2296-2300

Salvucci DD, Gray R (2004) A two-point visual control model of steering. Perception 33(10):1233-1248

Samuel S, Fisher DL (2015) Evaluation of the minimum forward roadway glance duration critical to latent hazard detection. In: Transportation research board 94th annual meeting (no. 15-1707)

Sarter NB, Woods DD (1995) How in the world did we ever get into that mode? Mode error and awareness in supervisory control. Hum Factors 37(1):5-19

Schneider W, Shiffrin RM (1977) Controlled and automatic human information processing: I. detection, search, and attention. Psychol Rev 84:1-66

Sebok A, Wickens CD (2017) Implementing lumberjacks and black swans into model-based tools to support human-automation interaction. Hum factors 59(2): 189-203

Senders JW, Kristofferson AB, Levison WH, Diettrich CW, Ward JL (1967) The attentional demand of automobile driving. In: Highway research record 195. Highway Research Board, National Research Council, Washington, DC, pp 15-33

Seppelt BD, Victor TW (2016) Potential solutions to human factors challenges in road vehicle automation. In: Meyer G, Beiker S (eds) Road vehicle automation 3. Lecture notes in mobility. Springer, Basel

Seppelt BD, Seaman S, Lee J, Angell LS, Mehler B, Reimer B (2017) Glass half-full: on-road glance metrics differentiate crashes from near-crashes in the 100-car data. Accid Anal Prev 107:48-62

Strand N, Nilsson J, Karlsson IM, Nilsson L (2014) Semi-automated versus highly automated driving in critical situations caused by automation failures. Transp Res Part F Traffic Psychol Behav 27:218-228

Subit D, Vézin P, Laporte S, Sandoz B (2017) Will automated driving technologies make today's effective restraint systems obsolete? Am J Public Health 107(10):1590-1592

Tivesten E, Dozza M (2014) Driving context and visual-manual phone tasks influence glance behavior in naturalistic driving. Transp Res Part F Traffic Psychol Behav 26:258-272

Tsimhoni O, Green P (2001) Visual demands of driving and the execution of display-intensive in-vehicle tasks. In: Proceedings of the human factors and ergonomics society 45 th annual meeting, pp $1586-1590$

Victor TW, Engström J, Harbluk JL (2008) Distraction assessment methods based on visual behaviour and event detection. In: Regan MA, Lee JD, Young KL (eds) Driver distraction: theory, effects, and mitigation. CRC Press, Taylor \& Francis Group, Boca Raton, FL, pp 135-165

Wickens CD (1992) Engineering psychology and human performance, 2nd edn. Harper Collins, New York

Wickens CD (2002) Multiple resources and performance prediction. Theor Issues Ergonom Sci 3(2):159-177

Wickens CD, Kessel C (1979) The effects of participatory mode and task workload on the detection of dynamic system failures. IEEE Trans Syst Man Cybern 9(1):24-34 
Wickens CD, Kessel C (1981) Failure detection in dynamic systems. In: Human detection and diagnosis of system failures. Springer, New York, pp 155-169

Zeeb K, Buchner A, Schrauf M (2015) What determines the take-over time? An integrated model approach of driver take-over after automated driving. Accid Anal Prev 78:212-221

Zeeb K, Härtel M, Buchner A, Schrauf M (2017) Why is steering not the same as braking? The impact of non-driving related tasks on lateral and longitudinal driver interventions during conditionally automated driving. Trans Res Part F Traffic Psychol Behav 50:65-79

Zuboff S (2010) In the age of the smart machine. In: Hanks C (ed) Technology and values: essential readings. Wiley-Blackwell, Oxford 